\title{
The Recognition of Roadbed Based on Fuzzy Etalons
}

\author{
Andrey G. Bronevich ${ }^{1}$, Bladimir I. Umanskiy ${ }^{2}$, Dmitriy A. Yakushev ${ }^{2}$ \\ ${ }^{1}$ National Research University Higher School of Economics, Moscow, Russia \\ ${ }^{2}$ JSC Intechgeotrans, Moscow, Russia
}

\begin{abstract}
In the paper we solve the problem of recognizing the roadbed using information about its possible typical designs. As shown on the paper typical designs can be adequately described by the set of fuzzy features, derived from the polygonal representation of the roadbed cross profile. The recognition is based on the minimization of a functional that takes into account how the polygon representation fits to the geometrical form of a measured profile and how its features obey the fuzzy restrictions. We propose to minimize this functional using probabilistic genetic algorithm. In the paper we also discuss the obtained results of the conducted experiments.
\end{abstract}

Keywords: Laser Scanning, Roadbed Classification, Polygonal Representations, Fuzzy Descriptions of Etalons.

\section{Introduction}

Nowadays there are several program systems that allow to monitor automatically railway roads. These systems give possibility to find defects linked with the track structure, clearance dimensions, rail cross profiles, etc. The full information about it can be found in [1-4]. The development of such systems is produced in two directions with the help of using new hardware or new software to attain higher monitoring speed and accuracy.

In the paper we consider the problem of railway profiles recognition that is used for detecting their defects, like non-normative breadth of ballast shoulder, nonnormative breadth of roadbed shoulder, places of oversized angles of slope. The review of the possible approaches to detecting such defects can be found in [2]). We assume that the input information for our methods are polygonal representations of measured cross profiles of the road bed. These polygonal representations can be statistically evaluated [5] by using clouds of points obtained by laser devices.

\section{Data description: cross profiles of roadbed}

The ideal cross profile of the roadbed can be represented as a polygon. Meanwhile, the real cross profile considerably differs from ideal, but it is possible to approximate its geometrical form by the sequence of straight segments. As we mention before, there is a problem of finding defects of the road bed, and it can be solved by comparing the measured profile with the normative profile according to the design decision. In
Russia there are special normative laws called «Constructional Norms and Rules, see [6] for example. There are some differences in solving such a problem linked with prior information in our disposal. The ideal situation is when the design decision is known. Then it is necessary to compare the etalon profile based on the decision design with the measured one to detect the set of possible defects. If the design decision is not known we need to recognize the measured profile using the set of all possible typical profiles. In this case we have the set of etalon profiles that correspond to different types of roadbed that can be classified as ditch cuts, embankments, tunnels, etc. Examples of such etalon profiles are shown on Fig. 1.

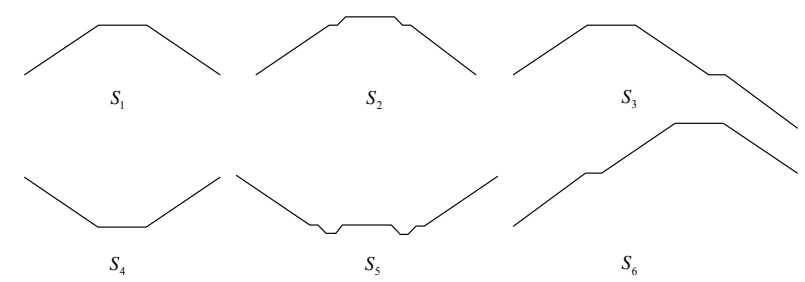

Fig. 1: Six etalon profiles.

The analysis of the cross profile of the roadbed assumes that we need to extract feature points, whose positions determine the basic characteristics of the roadbed. The feature points are points corresponding to edges upper and lower surfaces of embankment (ditch cut) of roadbed and ballast section. These points can be extracted by the statistical method of recovering profile described in Броневич 2011]. It is worth to mention that such points are extracted with different plausibility and reliability. It was experimentally checked that points describing the ballast section are extracted with the better degree of plausibility.

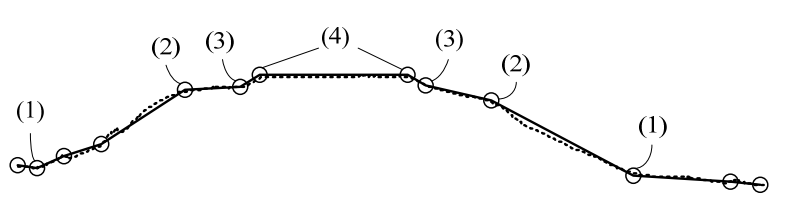

Fig. 2: The measured cross profile of the roadbed with extracted feature points.

The example of the measured profile is depicted on Fig. 2 with its polygonal representation. Each vertex of this representation can be interpreted as a feature point. One can see there edges of lower (1) and upper (2) surfaces, edges of lower (3) and upper (4) surfaces of the ballast section. 


\section{Fuzzy descriptions of etalon profiles}

Let us notice that the description of a etalon profile by a one polygonal representation is not adequate and allows us to extract only those structural elements of the roadbed, which have fixed dimensions, for example, the ballast section and the upper part of the roadbed. However, while some of parameters can be changed in sufficiently large intervals, e.g. the height of the embankment or the depth of a ditch cut, then such a model is not adequate. By this reason, we propose to describe etalon profiles with the help of fuzzy sets.

Let us consider the problem statement. Let us assume that profiles belonged to the same class can be described by polygonal representations $Y_{i}$ with the same number $M$ of points. Let us denote the set of all possible polygonal representations with the number $M$ of points by $\mathcal{Y}$. Then the fuzzy representation of an etalon class is a fuzzy set $S$ defined by a mapping $S: \mathcal{Y} \rightarrow[0,1]$. We will show next of how such a mapping (fuzzy set) can be constructed. Assume that each polygonal representation $Y \in \mathcal{Y}$ can be characterized by a set of features and any feature is a function $f_{i}: \mathcal{Y} \rightarrow \mathbb{R}$. In choosing a system of features one should use the normative documentation. Its analysis shows that features basically should be related to the parameters of polygon segments, in particular, the steepness of slants is characterized by tangent between segment and horizontal line, the ballast section is characterized by the segment length, etc. Thus, for any segment $\left(\mathbf{y}_{i}, \mathbf{y}_{i+1}\right), i=1, \ldots, M-1$, in the polygonal representation $Y=\left\{\mathbf{y}_{1}, \mathbf{y}_{2}, \ldots, \mathbf{y}_{M}\right\}$ we will put into correspondence the following features:

1) $\left(\mathbf{y}_{i+1}\right)_{x}-\left(\mathbf{y}_{i}\right)_{x}$ is the projection of the segment $\left(\mathbf{y}_{i}, \mathbf{y}_{i+1}\right)$ on $O x$ axis;

2) $\left(\mathbf{y}_{i+1}\right)_{y}-\left(\mathbf{y}_{i}\right)_{y}$ is the projection of the segment $\left(\mathbf{y}_{i}, \mathbf{y}_{i+1}\right)$ on Oy axis;

3) $\frac{\left(\mathbf{y}_{i+1}\right)_{y}-\left(\mathbf{y}_{i}\right)_{y}}{\left(\mathbf{y}_{i+1}\right)_{x}-\left(\mathbf{y}_{i}\right)_{x}}$ is the tangent of the segment slant.

We will assume next that the system of features has to define the polygonal representation uniquely. It means that for any segment it is sufficient to know two features, the rest one can be easily computed using known ones. After that based on experts opinions and using normative documentation it is possible to describe the possible values of features. This can be adequately described by fuzzy numbers. Let we want to describe the possible values of the ballast section length for a single track. According to the normative documentation this value should be equal to 2.3 meters. But for the real track this value can vary within [2.1,2.5], and the length outside this segment should be considered as defect of the track structure. The degree of such defect can be described by the fuzzy set $F_{i}: \mathbb{R} \rightarrow[0,1]$ depicted on Fig. 3.

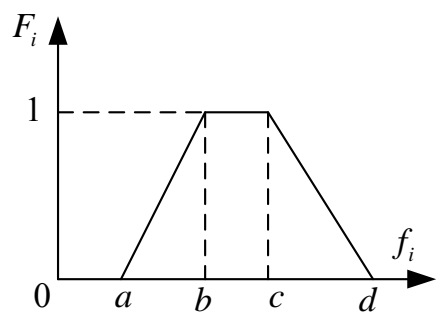

Fig. 3: Fuzzy set representation of possible feature values.

This is so called trapezoidal fuzzy number of $L-R$ type, and it can defined analytically as

$$
F_{i}(x)=\left\{\begin{array}{cc}
0, & x \in(-\infty, a] \cup[d,+\infty], \\
1, & x \in[b, c], \\
(x-a) /(b-a), & x \in(a, b), \\
(d-x) /(d-c), & x \in(c, d) .
\end{array}\right.
$$

Then for the feature «length of ballast section» we can choose $b=2.1, c=2.5$. The criticality of the feature value being outside the segment $[b, c]$ is described by parameters $a$ and $d$. For our example, we can take $a=1.9$ and $d=3$, since exceeding the admissible value for the length of ballast section is less dangerous than its dropping w.r.t. to lower bounds.

Let us assume now that for any etalon profile we have build the system $\left\{f_{1}, \ldots, f_{n}\right\}$ of features and possible values of each feature $f_{i}$ are described by the fuzzy number $F_{i}$. Then one among general approaches for choosing the mapping $S: \mathcal{Y} \rightarrow[0,1]$ is to use aggregation functions. By definition $\varphi:[0,1]^{n} \rightarrow[0,1]$ is an aggregation function if it obeys the following:

1) $\varphi(0, \ldots, 0)=0, \varphi(1, \ldots, 1)=1$ (norming);

2) $\varphi\left(x_{1}, \ldots, x_{n}\right) \leq \varphi\left(y_{1}, \ldots, y_{n}\right)$, если $x_{i} \leq y_{i}, i=1, \ldots, n$ (monotonicity).

For a given aggregation function $\varphi$ the mapping $S: \mathcal{Y} \rightarrow[0,1]$ is constructed by the formula:

$$
S(Y)=\varphi\left(F_{1}\left(f_{1}(Y)\right), \ldots, F_{n}\left(f_{n}(Y)\right)\right) .
$$

There are many approaches to choosing aggregation functions taking in account the interaction among features. If features don't interact each other, then the aggregation function is linear and it can be defined as

$$
\varphi\left(x_{1}, \ldots, x_{n}\right)=\sum_{i=1}^{n} w_{i} x_{i}
$$

where weights $w_{i}, i=1, \ldots, n$, are chosen non-negative and $\sum_{i=1}^{n} w_{i}=1$. Let us notice that features that defined parameters of segments in polygonal representations can be perceived as on-interactive, thus, it is possible to choose a linear function for aggregating them. The choice of $w_{i}$ is connected with the importance of these features for describing the roadbed. As a rule, the most 
important features are features which describe the upper road track as the width of the ballast section, the width of the basic ground, etc, but the features that describe the far located objects from the center line of the track can be considered as less important. It is also important to emphasize that precision of measurements of surfaces is decreased while we move away the center line of the track. It is explained by a chosen way of measurements, by possible shadows that appear often because lasers are not located very high about the ground surface, and by noises that can be induced by vegetation on the slants of the embankment. The optimal choice of these coefficients has to be based on expert opinions about processing of the system in a test mode.

\section{The recognition of cross profiles based on fuzzy etalons}

In this section we will consider that way of recognition that is closed to methods based on active contours [7]. Let us consider the problem statement. Given a polygonal representation $X=\left\{\mathbf{x}_{1}, \ldots, \mathbf{x}_{N}\right\}$ of the measured profile and the set of fuzzy set based descriptions of etalon profiles $\left\{S_{1}, \ldots, S_{K}\right\}$. Using this information we need to construct the decision functions $d\left(X, S_{k}\right)$, allowing us to make a decision " $X$ is belong to the class $S_{m}$ " if $d\left(X, S_{m}\right) \leq d\left(X, S_{k}\right)$ for all $k=1, \ldots, K$. While solving this problem we need to take in account that in fuzzy set based descriptions $S_{i}$ polygonal representations can differ by number of points in corresponding polygonal representations and also the number of points in a measured profile is also not fixed.

Let $X=\left\{\mathbf{x}_{1}, \ldots, \mathbf{x}_{N}\right\}$ be a measured profile of the roadbed and $S: \mathcal{Y} \rightarrow[0,1]$ be a fuzzy based description of the etalon profile, let us remind that $\mathcal{Y}$ is the set of all possible polygonal representations with $M$ points. In our comparison procedure we need to guarantee that comparing etalon and measured contours have the same number of points. It is possible to do this if we can generate polygonal representations in $\mathcal{Y}$ choosing points on polygonal representation $X$ lying on the segments of $X$. The set of all such possible polygonal representations will be denoted by $\mathcal{Y}_{X}$. Let us notice that each element of $\mathcal{Y}_{X}$ can be conceived as an approximation of $X$. The for any $Y \in \mathcal{Y}_{X}$ we can compute the value $S(Y)$; and if $S(Y)$ is larger than the representation $Y$ is more likely close to etalon one. Meanwhile, the construction of decision function has to take in consideration the quality of approximation. This property can measured using so called information measures of polygonal representations [8]. For this purpose for any $Y \in \mathcal{Y}_{X}$ introduce into consideration the auxiliary polygonal representation $Y \cup X$ that contains all points of the polygonal representation $Y$, where $Y=\left\{\mathbf{y}_{1}, \mathbf{y}_{2}, \ldots, \mathbf{y}_{M}\right\}$, and points of polygonal representation $X$, located between points $\mathbf{y}_{1}$ and $\mathbf{y}_{M}$. Thus, the profile $Y \underset{\leftarrow}{\cup}$ coincides with $X$ between points $\mathbf{y}_{1}$ and $\mathbf{y}_{M}$. Then we evaluate the quality of approximation using the value

$$
\left(\mu_{L}(Y \cup X)-\mu_{L}(Y)\right) / \mu_{L}(Y \cup X),
$$

where $\mu_{L}(Y \cup X), \mu_{L}(Y)$ are the lengths of the corresponding polygonal representations, in particular, $\mu_{L}(Y)=\sum_{i=2}^{M}\left|\mathbf{y}_{i}-\mathbf{y}_{i-1}\right|$. Let us notice that the introduced estimate of approximation quality is equal to zero if profile $Y$ coincides with profile $X$ between points $\mathbf{y}_{1}$ and $\mathbf{y}_{M}$. Thus, we have two characteristics of the polygonal representation $Y$ : the value $S(Y)$ shows how profile $Y$ is close to the etalon one and $\left(\mu_{L}(Y \cup X)-\mu_{L}(Y)\right) / \mu_{L}(Y \cup X)$ defines the quality of approximation. To get the resulting criterion, we get their linear combination:

$$
-a S(Y)+b \frac{\mu_{L}(Y \underset{\leftarrow}{\cup})-\mu_{L}(Y)}{\mu_{L}(Y \cup X)},
$$

where $a>0$ and $b>0$. Then the above criterion is less when $Y$ is better approximates $X$ and when $Y$ is closer to the etalon contour. Then the choice of $Y \in \mathcal{Y}_{X}$ that close to the etalon contour with a good approximation of initial contour $X$ can be produced by solving the following optimization problem:

$$
d(X, S)=\inf _{Y \in \mathcal{Y}_{X}}\left(-a S(Y)+b \frac{\mu_{L}(Y \underset{\leftarrow}{\cup} X)-\mu_{L}(Y)}{\mu_{L}(Y \cup X)}\right) .
$$

Let us notice that analogous optimization problems are solved in recognition methods based on active contours. In such optimization problems two functionals are used and called inner and outer energies of a contour. The outer energy characterizes the quality of approximation, and the inner energy describes the restrictions on the choice of contour.

\section{The computation of decision functions based on probabilistic genetic algorithm}

Clearly, the computing decision functions is sufficiently hard optimization problem with many local minima, in addition, the differential characteristics of the optimized functional is not good. This does not allows us to use classical optimization methods. Therefore, in this section we introduce the genetic probabilistic algorithm that produces the probabilistic search of local minima.

Assume that for the etalon $S$ is chosen the complete and non-redundant system $\left\{f_{1}, \ldots, f_{n}\right\}$ of features, that is each profile can be uniquely recovered by values of $f_{1}, \ldots, f_{n}$. Additionally, we have the fuzzy restrictions on the values of features, described by fuzzy sets 
$F_{1}, \ldots, F_{n}$. Then the probabilistic genetic algorithm for computing $d(X, S)$ is described as follows.

Initial data: to minimize functional:

$$
\Phi(Y)=-a S(Y)+b \frac{\mu_{L}(Y \underset{\leftarrow}{\cup})-\mu_{L}(Y)}{\mu_{L}(Y \cup X)} .
$$

We have in our disposal: 1) the measured profile $X$ and the etalon profile $S$ defined by the system $\left\{f_{1}, \ldots, f_{n}\right\}$ of features and the fuzzy restrictions $\left\{F_{1}, \ldots, F_{n}\right\}$. For the profile $X$ and each profile $Y \in \mathcal{Y}$ we know the location of the center line of the track.

Step 0. Generate the sample $\left\{Y_{1}, \ldots, Y_{N}\right\}$ that satisfies fuzzy restrictions $F_{1}, \ldots, F_{n}$, in a way that the center line of the track for profiles $X$ and $Y_{1}, \ldots, Y_{N}$ have the same location.

Step 1. Profiles $Y_{1}, \ldots, Y_{N}$ are projected on profile $X$. The sense of this step is in the following. Obviously, each $Y_{i}$ does not "lie" on the profile $X$. In order to the above property is fulfilled each profile $Y_{i}$ is exchanged to its projection $\operatorname{Pr}_{X} Y_{i}$. This operation is produced such that coordinates of points along $O x$ of $Y_{i}$ and $\operatorname{Pr}_{X} Y_{i}$ are chosen the same, but points $\operatorname{Pr}_{X} Y_{i}$ should belong to contour $X$.

Step 2. Compute values $\Phi\left(\operatorname{Pr}_{X} Y_{i}\right), i=1, \ldots, N$, and order the sample such that $\Phi\left(\operatorname{Pr}_{X} Y_{i_{1}}\right) \leq \Phi\left(\operatorname{Pr}_{X} Y_{i_{2}}\right) \leq \ldots$ $\leq \Phi\left(\operatorname{Pr}_{X} Y_{i_{N}}\right)$.

Step 3. Truncate the sample: in new sample we have profiles $\operatorname{Pr}_{X} Y_{i_{1}}, \operatorname{Pr}_{X} Y_{i_{2}}, \ldots Y_{i_{N_{1}}}$, where $N_{1}\left(N_{1}<N\right)$ where is the parameter of the algorithm. (We can take $N:=100$ и $N_{1}:=50$.)

Step 4. The evaluation of feature distributions for $f_{i}$, $i=1, \ldots, n$, produced truncated sample по $\operatorname{Pr}_{X} Y_{i_{1}}, \operatorname{Pr}_{X} Y_{i_{2}}, \ldots Y_{i_{N_{1}}}$. It is possible to assume that $f_{i}$ are independent random variables and they are distributed normally.

Step 5. Check the condition of convergence: the value $d(X, S)$ is computed if the evaluated distribution of each feature $f_{i}$, obeying the fuzzy restriction $F_{i}$, has been converged to the spotted distribution. If this condition is not fulfilled, then go to Step 6 .

Step 6. Generate the sample $Y_{1}, \ldots, Y_{N}$ according to evaluated feature distributions of $f_{i}, i=1, \ldots, n$. Go to Step 1.

Let us explain in detail some steps of the proposed algorithm.

On Step 0 it is required to generate the sample $\left\{Y_{1}, \ldots, Y_{N}\right\}$ that satisfies fuzzy restrictions $F_{1}, \ldots, F_{n}$. To do this we use the probabilistic interpretation of fuzzy sets $F_{1}, \ldots, F_{n}$. In this interpretation any fuzzy set $F_{i}$ is identified with the random set as follows. We consider non-strict cuts $\left(F_{i}\right)_{t}=\left\{x \in \mathbb{R} \mid F_{i}(x) \geq t\right\}, t \in(0,1]$ of
$F_{i}$ and assume that $t$ is a random variable uniformly distributed on $(0,1]$. After that for a random choice within the random set $F_{i}$ one can use the probabilistic model of the second order, i.e. we assume that $f_{i}$ is a random variable uniformly distributed within the interval $F_{i}$. Let us consider how to describe the distribution of the random variable $f_{i}$ if $F_{i}$ is a trapezoidal fuzzy number of $L-R$ type depicted on Fig. 3. In this case

$$
\left(F_{i}\right)_{t}=[a+(b-a) t, d-(d-c) t],
$$

and density $\pi_{t}$ of uniformly distributed $f_{i}$ for fixed $t$ is computed as

$$
\pi_{t}(x)=\frac{1}{(d-a)-((d-a)-(c-b)) t}
$$

for $x \in[a+(b-a) t, d-(d-c) t]$, and $\pi_{t}(x)=0$ otherwise. Then density $\pi$ of the random variable $f_{i}$ can be computed as

$$
\pi(x)=\int_{0}^{1} \pi_{t}(x) d t
$$

After integrating we get

1) $\pi(x)=\frac{\ln (d-a)-\ln A(x)}{(d-a)-(c-b)}$, where

$A(x)=(d-a)-((d-a)-(c-b)) \frac{x-a}{b-a}$ and $x \in[a, b)$;

2) $\pi(x)=\frac{\ln (d-a)-\ln (c-b)}{(d-a)-(c-b)}, x \in[b, c]$;

3) $\pi(x)=\frac{\ln (d-a)-\ln B(x)}{(d-a)-(c-b)}$, where

$B(x)=(d-a)-((d-a)-(c-b)) \frac{d-x}{d-c}$ and $x \in(c, d]$;

4) $\pi(x)=0, x \notin[a, d]$.

On Step 5 we need to check the convergence to the spotted distribution for each feature $f_{i}$ satisfying the fuzzy restriction $F_{i}$. On this step we have the sample $\left\{\operatorname{Pr}_{X} Y_{i_{1}}, \operatorname{Pr}_{X} Y_{i_{2}}, \ldots \operatorname{Pr}_{X} Y_{i_{N_{1}}}\right\}$. Using this sample we compute the values of $f_{i}$ :

$$
\left\{f_{i}\left(\operatorname{Pr}_{X} Y_{i_{1}}\right), f_{i}\left(\operatorname{Pr}_{X} Y_{i_{2}}\right), \ldots f_{i}\left(\operatorname{Pr}_{X} Y_{i_{N_{1}}}\right)\right\}
$$

If at least $f_{i}\left(\operatorname{Pr}_{X} Y_{i_{k}}\right) \in\left(F_{i}\right)_{1}$ for one $Y_{i_{k}}$, then we suppose that the feature $f_{i}$ obeys fuzzy restrictions. If we approximate $f_{i}$ by the normal distribution, then we can compute the estimate $\hat{\sigma}\left[f_{i}\right]$ of standard deviation using the sample $\left\{f_{i}\left(\operatorname{Pr}_{X} Y_{i_{1}}\right), f_{i}\left(\operatorname{Pr}_{X} Y_{i_{2}}\right), \ldots f_{i}\left(\operatorname{Pr}_{X} Y_{i_{N_{1}}}\right)\right\}$. Then 
the convergence of estimated probability distribution to the spotted distribution can be checked using the inequality $\hat{\sigma}\left[f_{i}\right]<\varepsilon_{i}$, where $\varepsilon_{i}$ is the parameter of the algorithm.

\section{Experimental results and conclusions}

The proposed method for recognizing cross roadbed profiles has been tested on real and simulated data. Tests on simulated data has shown that the method sometimes does not recognize correctly parts of the roadbed located far from the center line of the road track, like ditches for withdrawal water like on Fig. 5. But for real data (see Fig. 4) it seems it is not so crucial because the precision is not so good for recognizing such details using scanning data, where it is possible to have good results by the proposed method for recognizing upper track construction.

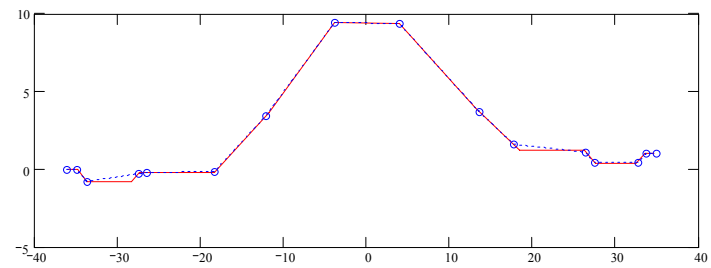

Fig. 4: Red line: initial profile $X$, Blue line: the projection of constructed etalon profile $Y$.

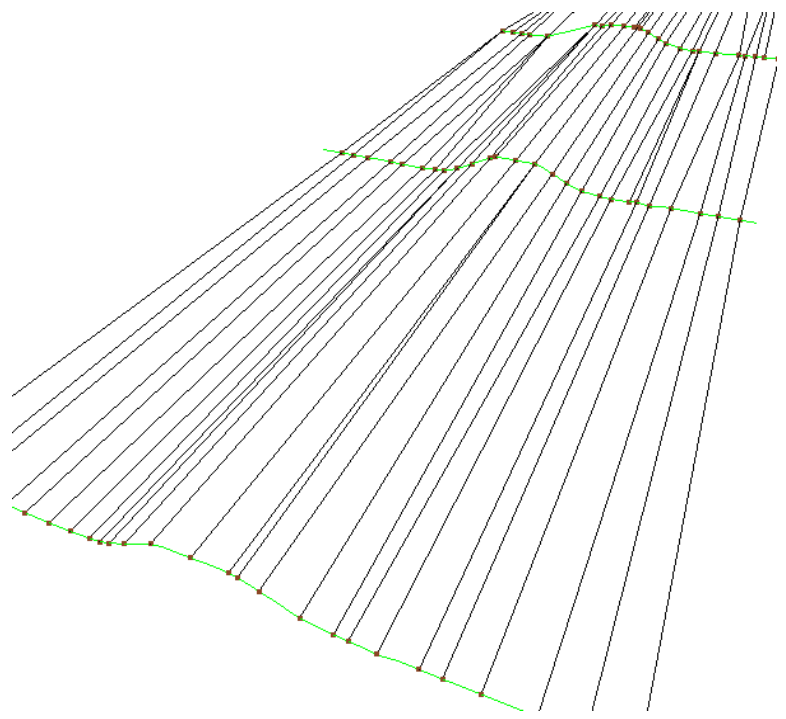

Fig. 5: The recovered surface of the roadbed using the scanning data.

As shown experimental results the effective realization of the proposed method depends on the optimization procedure. The features appear to be dependent and the algorithm tries to recognize points of the polygonal representation starting from the center line of the track. If during this recognizing some points are classified not correctly then it recognizes not correctly the next points. Therefore, the next research can be done for improving the optimization procedure which can be realized like in recognizing algorithms based different pyramid representations.

\section{Acknowledgment}

This work was supported by the grant 11-07-00591 of RFBR (Russian Foundation for Basic Research).

\section{References}

[1] H. Trinh, N. Haas, Y. Li, C. Otto and S. Pankanti, Enhanced rail component detection and consolidation for rail track inspection. In: Proc. IEEE Workshop on the Applications of Computer Vision, pages 289-295, 2012.

[2] Babenko, P.: Visual inspection of railroad tracks. In $\mathrm{PhD}$ Thesis, http ://server.cs.ucf.edu/vision/papers/ theses/BabenkoPavel.pdf (2009).

[3] A. Berry, B. Nejikovsky, X. Gilbert and A. Jajaddini, High speed video inspection of joint bars using advanced image collection and processing techniques. In Proc. of World Congress on Railway Research (2008).

[4] Machine Vision Inspection of Railroad Track. Technical report, NEXTRANS Project No.0281Y02 (2011)

[5] A.G. Bronevich, A.N. Karkishchenko and V.I. Umanskiy, Statistical method of restoring the profile from laser scanning data. Digital Signal Processing, 4: 42-49, 2011. (in Russian)

[6] Constructional Norms and Rules 32-01-95. The railways of $1520 \mathrm{~mm}$ (1995) (in Russian)

[7] M. Kass, A. Witkin and D. Terzopoulos, Snakes: active contour models. International Journal of Computer Vision, 1: 321-331, 1988.

[8] A.G. Bronevich and A.E. Lepskiy, Geometrical fuzzy measures in image processing and pattern recognition // In Proc. of the 10th IFSA World Congress, Istanbul, Turkey, pages 151-154, 2003. 